\title{
INVERSE SEMIGROUPS WITH IDEMPOTENTS DUALLY WELL-ORDERED
}

\author{
A. CLEMENT and F. PASTIJN
}

(Received 17 May 1982)

Communicated by T. E. Hall

\begin{abstract}
All inverse semigroups with idempotents dually well-ordered may be constructed inductively. The techniques involved are the constructions of ordinal sums, direct limits and Bruck-Reilly extensions. 1980 Mathematics subject classification (Amer. Math. Soc.): 20 M 10, 03 E 10.
\end{abstract}

\section{Introduction}

We use the terminology and the results of Howie [3] and Sierpiński [12]. The axiom of choice will be assumed throughout.

A study of inverse semigroups with idempotents dually well-ordered can be motivated by the findings of Feller and Gantos [1]. They may also be studied within the context of the investigations by Megyesi and Pollák ([5], [6], [7]) concerning principal ideal semigroups. Recall that a principal ideal semigroup is a semigroup where the left, right and two-sided ideals are all principal, or equivalently, it is a semigroup for which the posets of left, right and two-sided ideals are dually well-ordered chains. In a [simple] principal ideal semigroup, the set of regular elements - if non-empty - constitutes a [simple] inverse semigroup with idempotents dually well-ordered. In fact, a principal ideal semigroup is regular if and only if it is an inverse semigroup with idempotents dually well-ordered.

Particular structure theorems for inverse semigroups with idempotents dually well-ordered were given by Hogan [2], Kočin [4], Munn [8], Reilly [11], and Warne ([13], [14]). 


\section{Main results}

Let $\delta$ be any ordinal. An inverse semigroup $S$ will be called a $\delta$-regular semigroup if the set $E_{S}$ of idempotents of $S$ constitutes a chain whose order type is $\bar{E}_{S}=\delta^{*}$.

Recall that an inverse semigroup $S$ is called a fundamental inverse semigroup if the greatest idempotent-separating congruence on $S$ is the identity relation. Let us consider a fundamental inverse semigroup $S$ whose idempotents form a chain $E_{S}$. Then Green's equivalence relation $g$ is the least semilattice congruence on $S$, and $S$ is a chain $S / \mathcal{G}$ of its $g$-classes, which are all simple inverse semigroups. Each $g$-class is the disjoint union of $\mathscr{D}$-classes which all constitute bisimple inverse semigroups. In general it is not straightforward how to describe $S$ as a chain composition of its $G$-classes. There is however an important instance in which things simplify. Indeed, let us consider the case where the principal ideals of $E_{S}$ each have a trivial automorphism group. Since $S$ is fundamental, one can embed $S$ isomorphically into the Munn semigroup $T_{E_{S}}$ (see, for example, Howie [3]). It follows that $S$ must be combinatorial (= HG-trivial), and for any $a, b \in S$, with $J_{a}<J_{b}$ in $S / g$, we have $a b=b a=a$. The situation described here is satisfied whenever $E_{S}$ is a dually well-ordered chain. We thus have the following.

TheOREM 1. Let $\delta=\Sigma_{\xi<\alpha} \alpha_{\xi}$ be an ordinal such that for each $\xi<\alpha, S_{\xi}$ is a combinatorial simple $\alpha_{\xi}$-regular semigroup, with $S_{\xi} \cap S_{\eta}=\varnothing$ if $\xi \neq \eta$. On $S=$ $\cup_{\xi<\alpha} S_{\xi}$ define a multiplication by the following. If $a \in S_{\xi}, b \in S_{\eta}$, then $a b$ coincides with the product of $a$ and $b$ already defined in $S_{\xi}$ if $\xi=\eta$, whereas $a b=a$ if $\xi>\eta$ and $a b=b$ if $\eta>\xi$. Then $S$ is a fundamental $\delta$-regular semigroup.

Conversely, every fundamental $\delta$-regular semigroup can be so obtained.

COROLlary 2. Let $\delta=\Sigma_{\xi<\alpha} \alpha_{\xi}$ be an ordinal such that for each $\xi<\alpha, S_{\xi}$ is a simple $\alpha_{\xi}$-regular semigroup, with $S_{\xi} \cap S_{\eta}=\varnothing$ if $\xi \neq \eta$. For every $\xi<\eta<\alpha$, let $\phi_{\xi, \eta}$ be a homomorphism of $S_{\xi}$ into the group of units of $S_{\eta}$, such that $\phi_{\xi, \eta} \phi_{\eta, \zeta}=\phi_{\xi, \zeta}$ whenever $\xi<\eta<\zeta<\alpha$. For each $\xi<\alpha$, let $\phi_{\xi, \xi}$ be the identity transformation on $S_{\xi}$. Let $S$ be the strong chain of the semigroups in the system

$$
\left(\alpha ;\left\{S_{\xi} \mid \xi<\alpha\right\} ;\left\{\phi_{\xi, \eta} \mid \xi \leqslant \eta<\alpha\right\}\right) .
$$

Then $S$ is a $\delta$-regular semigroup.

Conversely, every $\delta$-regular semigroup can be so obtained.

Proof. The direct part can be verified without difficulty.

Let us conversely suppose that $S$ is a $\delta$-regular semigroup. Since the principal ideals of $E_{S}$ have trivial automorphism groups, Green's relation $\mathcal{K}$ is a congruence 
relation on $S$ (see also Theorem 5 in Megyesi and Pollák [5]). Therefore $S / \mathcal{H}$ is a fundamental $\delta$-regular semigroup, and we can apply Theorem 1 . The results of Theorem 1 guarantee that we can write $S$ as a chain $\alpha$ of simple $\alpha_{\xi}$-regular semigroups $S_{\xi}, \xi<\alpha$, with $\delta=\sum_{\xi<\alpha} \alpha_{\xi}$. Further, if $\xi<\eta$, and if $1_{\eta}$ denotes the identity element of $S_{\eta}$, then the mapping

$$
\phi_{\xi, \eta}: S_{\xi} \rightarrow S_{\eta}, a \rightarrow a 1_{\eta}
$$

is a homomorphism of $S_{\xi}$ into the group of units of $S_{\eta}$. As a result we obtain a system (1), and one easily shows that $S$ is the sum of this system.

If the semigroup $S$ is obtained in the way described in Corollary 2, then we shall say that $S$ is the ordinal sum of the system (1).

We exemplify Theorem 1 by describing the Munn semigroup $T_{E}$ of a chain $E$ whose order type $\bar{E}$ is the dual $\delta^{*}$ of an ordinal $\delta$. One may identify $T_{E}$ with the inverse semigroup consisting of the isomorphisms among principal filters of $\delta$ (where $\delta$ stands for the well-ordered chain of ordinals that are less than $\delta$ ). The latter inverse semigroup will be denoted by $T_{\delta}$. Remark that $T_{\omega}$ is the bicyclic semigroup, whereas $T_{\omega^{n}}$ ( $n$ a positive integer) is Warne's $n$-dimensional bicyclic semigroup [13], and $T_{\omega^{\alpha}}$ ( $\alpha$ any ordinal) is the $\alpha$-bicyclic semigroup in Hogan [2] and Megyesi and Pollák [7]. Let $\xi$ and $\eta$ be ordinals such that $\delta=\xi+\tau=\eta+\tau$ for some ordinal $\tau$. Then the principal filter generated by $\xi$ is isomorphic to the principal filter generated by $\eta$ : the two filters are of order type $\tau$. We denote by $\left(\begin{array}{l}\xi \\ \eta\end{array}\right)$ the unique isomorphism of the principal filter generated by $\xi$ onto the principal filter generated by $\eta$. Thus,

$$
(\xi+\kappa)\left(\begin{array}{l}
\xi \\
\eta
\end{array}\right)=\eta+\kappa, \quad \kappa<\tau
$$

The inverse of $\left(\begin{array}{l}\xi \\ \eta\end{array}\right)$ in $T_{\delta}$ is $\left(\begin{array}{l}\eta \\ \xi\end{array}\right)$. Clearly $T_{\delta}$ precisely consists of the elements $\left(\begin{array}{l}\xi \\ \eta\end{array}\right)$ where $\delta=\xi+\tau=\eta+\tau$ for some ordinal $\tau$, and the multiplication in $T_{\delta}$ is given by

$$
\left(\begin{array}{l}
\xi \\
\eta
\end{array}\right)\left(\begin{array}{l}
\xi^{\prime} \\
\eta^{\prime}
\end{array}\right)=\left(\begin{array}{c}
\xi+\left[\xi^{\prime}-\eta\right] \\
\eta^{\prime}+\left[\eta-\xi^{\prime}\right]
\end{array}\right),
$$

where for any ordinals $\rho, \sigma$

$$
[\rho-\sigma]= \begin{cases}\rho-\sigma & \text { if } \sigma \leqslant \rho \\ 0 & \text { otherwise }\end{cases}
$$

(we use the notation of Megyesi and Pollák [7]).

Recall that for any ordinal $\delta$ there exists a unique decomposition $\delta=\delta_{1}$ $+\cdots+\delta_{k}$ ( $k$ a positive integer), where $\delta_{1} \geqslant \cdots \geqslant \delta_{k}$ is a finite nonincreasing sequence of prime ( $=$ indecomposable) ordinals. This decomposition is called the normal expansion of $\delta$. 
THEOREM 3. Let $\delta$ be an ordinal, and $\delta=\delta_{1}+\cdots+\delta_{k}$ its normal expansion. Then $T_{\delta}$ is a $k$-chain of the bisimple combinatorial $\delta_{i}$-regular semigroups $T_{i}$, $i=1, \ldots, k$. For each $i=1, \ldots, k, T_{i}$ is isomorphic to $T_{\delta_{i}}$.

Proof. Let $\xi, \eta$ be ordinals such that $\delta=\xi+\tau=\eta+\tau$. The ordinal $\tau$ must be of the form $\delta_{i}+\cdots+\delta_{k}$ for some $1 \leqslant i \leqslant k$. Putting

$$
T_{i}=\left\{\left(\begin{array}{l}
\xi \\
\eta
\end{array}\right) \mid \delta=\xi+\delta_{i}+\cdots+\delta_{k}=\eta+\delta_{i}+\cdots+\delta_{k}\right\}
$$

for $i=1, \ldots, k$, we obtain a partitioning $T_{\delta}=\cup_{1<i<k} T_{i}$. Let $\left(\begin{array}{l}\xi \\ \eta\end{array}\right),\left(\begin{array}{l}\xi^{\prime} \\ \eta^{\prime}\end{array}\right) \in T_{i}$ for some $1 \leqslant i \leqslant k$. Then

$$
\left(\begin{array}{c}
\xi \\
\eta
\end{array}\right) \Re\left(\begin{array}{c}
\xi \\
\eta^{\prime}
\end{array}\right) \mathcal{L}\left(\begin{array}{c}
\xi^{\prime} \\
\eta^{\prime}
\end{array}\right) \Re\left(\begin{array}{l}
\xi^{\prime} \\
\eta
\end{array}\right) \mathcal{E}\left(\begin{array}{l}
\xi \\
\eta
\end{array}\right)
$$

in $T_{\delta}$. Consequently $T_{i}$ is contained in a $\mathscr{D}$-class. Further, if $\left(\begin{array}{l}\xi \\ \eta\end{array}\right) \in T_{i},\left(\begin{array}{l}\xi^{\prime} \\ \eta^{\prime}\end{array}\right) \in T_{j}$, $i<j$, then $\left(\begin{array}{l}\xi \\ \eta\end{array}\right)\left(\begin{array}{l}\xi^{\prime} \\ \eta^{\prime}\end{array}\right)=\left(\begin{array}{l}\xi^{\prime} \\ \eta^{\prime}\end{array}\right)\left(\begin{array}{l}\xi \\ \eta\end{array}\right)=\left(\begin{array}{l}\xi^{\prime} \\ \eta^{\prime}\end{array}\right)$. Thus elements belonging to different components in the partitioning $\cup_{1<i<k} T_{i}$ cannot be $\mathcal{g}$-related. We see that $\mathcal{g}=\mathscr{D}$ in $T_{\delta}$, and that the $T_{i}, i=1, \ldots, k$, constitute the $k$ D-classes of $T_{\delta} ; T_{\delta}$ is a $k$-chain of these $\mathscr{D}$-classes.

The D-classes $T_{i}, i=1, \ldots, k$, form bisimple inverse semigroups (see the remark made before Theorem 1). $T_{\delta}$ is combinatorial since well-ordered chains have a trivial automorphism group. Thus the $T_{i}, i=1, \ldots, k$ are combinatorial as well. The idempotents of $T_{i}$ are of the form $(\xi)$, with $\xi<\delta_{1}$ if $i=1$, or $\delta_{1}+\cdots+\delta_{i-1}$ $\leqslant \xi<\delta_{1}+\cdots+\delta_{i}$ otherwise. Therefore $T_{i}$ is a $\delta_{i}$-regular semigroup.

The mapping

$$
T_{1} \rightarrow T_{\delta_{1}}, \quad\left(\begin{array}{l}
\xi \\
\eta
\end{array}\right) \rightarrow\left(\begin{array}{l}
\xi \\
\eta
\end{array}\right)
$$

is easily seen to be an isomorphism of $T_{1}$ onto $T_{\delta_{1}}$, whereas in the case $1<i \leqslant k$,

$$
T_{i} \rightarrow T_{\delta_{i}}, \quad\left(\begin{array}{l}
\xi \\
\eta
\end{array}\right) \rightarrow\left(\begin{array}{l}
\xi-\left(\delta_{1}+\cdots+\delta_{i-1}\right) \\
\eta-\left(\delta_{1}+\cdots+\delta_{i-1}\right)
\end{array}\right)
$$

is an isomorphism of $T_{i}$ onto $T_{\delta_{i}}$.

COROLlary 4. Let $E$ be a chain such that $\bar{E}^{*}$ is an ordinal. In the Munn semigroup $T_{E}, \mathcal{G}$ and $\mathscr{D}$ coincide. The number of $\mathscr{D}$-classes in $T_{E}$ is finite. It is the number of terms in the normal expansion of $\bar{E}^{*}$.

Corollary 5 (Hogan [2], Munn [9], White [15]). If $S$ is a simple $\delta$-regular semigroup, then $\delta$ is a prime ordinal. If $E$ is a chain such that $\bar{E}^{*}=\delta$ is a prime ordinal, then $T_{E}$ is a bisimple $\delta$-regular semigroup. 
Theorem 1 and Corollary 2 show that the problem of describing the structure of [fundamental] inverse semigroups with idempotents dually well-ordered can be reduced to the case of simple [fundamental] inverse semigroups with idempotents dually well-ordered. Therefore we shall from now on concentrate on simple $\delta$-regular semigroups. From Corollary 5 we know that $\delta$ must then be a prime ordinal, that is, $\delta=\omega^{\alpha}$ for some ordinal $\alpha$ (well-defined by $\delta$ ). The aim of our considerations will be to construct simple $\omega^{\alpha}$-regular semigroups in terms of $\xi$-regular semigroups, with $\xi<\omega^{\alpha}$. This will enable us to construct inductively all inverse semigroups with idempotents dually well-ordered.

If $T$ is a $\delta$-regular semigroup and $\theta$ an endomorphism of $T$ into the unit group of $T$, then one can consider the Bruck-Reilly extension $B R(T, \theta)$ of $T$ determined by $\theta$. This inverse semigroup $B R(T, \theta)$ must be a simple $\delta \omega$-regular semigroup (see for example III.2 of Petrich [10]). Thus, any $\delta$-regular semigroup can be embedded into a simple $\delta \omega$-regular semigroup. Note that $B R(T, \theta)$ is fundamental if and only if $T$ is fundamental. If this is the case, then $\theta$ is simply the constant mapping of $T$ onto the identity of $T$. The following characterizes the inverse semigroups with idempotents dually well-ordered which are obtained by considering Bruck-Reilly extensions.

THeOREM 6. Let $S$ be a $\delta$-regular semigroup, with $E_{S}=\left\{e_{\xi} \mid \xi<\delta\right\}$, where $e_{\xi}<e_{\eta}$ in $E_{S}$ if and only if $\eta<\xi$. Then $S$ is a Bruck-Reilly extension $B R(T, \theta)$ if and only if the following conditions are satisfied:

(i) $\delta=\omega^{\alpha+1}$ for some $\alpha$,

(ii) there exists $a \omega^{\alpha} \leqslant \gamma<\omega^{\alpha+1}$ such that $e_{0} \mathscr{D} e_{\gamma}$ and such that the elements $x \in S$ for which $e_{\gamma}<\left(x x^{-1}\right)\left(x^{-1} x\right)$ form a subsemigroup of $S$.

Proof. Let $S=B R(T, \theta)$ for some inverse semigroup $T$, and for some appropriate endomorphism $\theta$ of $T$. From the fact that $S$ is a $\delta$-regular semigroup it follows that $T$ is an inverse semigroup with idempotents dually well-ordered. In other words, $T$ is a $\gamma$-regular semigroup for some ordinal $\gamma$, where $\delta=\gamma \omega$. Let $\omega^{\alpha}$ be the first term in the normal expansion of $\gamma$. Then $\delta=\omega^{\alpha+1}$, and so (i) is satisfied. Let us denote the set of idempotents of $T$ by $\left\{f_{\zeta} \mid \zeta<\gamma\right\}$, where $f_{\zeta}<f_{\tau}$ in $E_{T}$ if and only if $\tau<\zeta$. The idempotents of $S$ are then of the form

$$
e_{\gamma n+\zeta}=\left(n, f_{\zeta}, n\right), \quad n \in N, \zeta<\gamma,
$$

and one sees that (ii) is satisfied.

Let us conversely suppose that $S$ satisfies (i) and (ii). Let $T$ be the subsemigroup of $S$ which is given by (ii). Since $T$ is clearly closed with respect to the taking of inverses, we have that $T$ is an inverse subsemigroup of $S$. Consequently, $T$ is a $\gamma$-regular subsemigroup of $S$. Let $a$ be an element of $S$ for which $a a^{-1}=e_{0}$ and $a^{-1} a=e_{\gamma}$. 
Let $x \in S$, with $x x^{-1}=e_{\xi}$ and $x^{-1} x=e_{\eta}$. Then

$$
S \rightarrow T_{\delta}, \quad x \rightarrow\left(\begin{array}{c}
\xi \\
\eta
\end{array}\right)
$$

is a representation of $S$ which is equivalent to the Munn representation. In particular, if $x \in T$, then $\left(\begin{array}{l}\xi \\ \eta\end{array}\right)$ must fix $\gamma$ since $T$ forms a subsemigroup, and since $T_{\delta}$ is combinatorial. In this case we must have $\left(\begin{array}{l}\xi \\ \eta\end{array}\right)\left(\begin{array}{l}\gamma \\ \gamma\end{array}\right)=\left(\begin{array}{l}\gamma \\ \gamma\end{array}\right)\left(\begin{array}{l}\xi \\ \eta\end{array}\right)=\left(\begin{array}{c}\gamma \\ \gamma\end{array}\right)$, and consequently

$$
e_{\gamma} x \mathcal{H} e_{\gamma} \mathcal{H} x e_{\gamma} \text { for all } x \in T
$$

It follows that

$$
\theta: T \rightarrow H_{e_{0}}, \quad x \rightarrow a x a^{-1}
$$

is an endomorphism of $T$ into its group of units.

For $m, n \in N$, let $S_{m, n}$ consist of the elements $x$ of $S$ for which $e_{\gamma m} \geqslant x x^{-1}>$ $e_{\gamma(m+1)}$ and $e_{\gamma n} \geqslant x^{-1} x>e_{\gamma(n+1)}$. Then $S=\cup_{m, n \in N} S_{m, n}$ yields a partitioning of $S$. Remark that $T=S_{0,0}$. The mapping $T \rightarrow S_{m, n}, x \rightarrow a^{-m} x a^{n}$ is a bijection of $T$ onto $S_{m, n}$, and the mapping $S_{m, n} \rightarrow T, y \rightarrow a^{m} y a^{-n}$ is its inverse. For this reason

$$
\psi: S \rightarrow B R(T, \theta), \quad a^{-m} x a^{n} \rightarrow(m, x, n), \quad m, n \in N, x \in T,
$$

is a well-defined bijection of $S$ onto $B R(T, \theta)$. It is easy to show that $\psi$ is in fact an isomorphism.

THEOREM 7. Let $S$ be a simple $\omega^{\alpha}$-regular semigroup, with $\alpha$ a limit ordinal. Then there exists a well-ordered system

$$
\left(\beta ;\left\{S_{\xi} \mid \xi<\beta\right\} ;\left\{\phi_{\xi, n} \mid \xi \leqslant \eta<\beta\right\}\right)
$$

of simple $\omega^{\alpha_{\xi}+1}$-semigroups $S_{\xi}, \xi<\beta$, where

(i) for $\xi<\beta, S_{\xi}=B R\left(T_{\xi}, \theta_{\xi}\right)$ is a Bruck-Reilly extension of $a\left(\omega^{\alpha_{\xi}}+\delta_{\xi}\right)$-regular semigroup $T_{\xi}$, with $\delta_{\xi}<\omega^{\alpha_{\xi}+1}$,

(ii) $\alpha=\lim _{\xi<\beta}\left(\alpha_{\xi}+1\right)$,

(iii) for $\xi \leqslant \eta<\beta, \phi_{\xi, \eta}$ is a monomorphism of $S_{\xi}$ into $S_{\eta}$, such that $S$ is the direct limit of the system (11).

Conversely, if the well-ordered system (11) satisfies the above conditions (i), (ii) and (iii), then its direct limit is a simple $\omega^{\alpha}$-regular semigroup.

Proof. The proof of the converse part is routine, and is left to the reader. We now proceed to show the direct part.

Let $\left\{e_{\zeta} \mid \zeta<\omega^{\alpha}\right\}$ be the set of idempotents of $S$, where $e_{\zeta}<e_{\eta}$ in $E_{S}$ if $\eta<\zeta$. Let $A$ be a set of ordinals, where $\kappa \in A$ if and only if there exists a $\omega^{\kappa} \leqslant \gamma<\omega^{\kappa+1}$ such that $e_{0} \mathscr{D} e_{\gamma}$ in $S$. Let $\beta$ be the order type of the chain $A$. We shall denote the 
chain $A$ by $A=\left\{\alpha_{\xi} \mid \xi<\beta\right\}$. We have $\lim _{\xi<\beta} \alpha_{\xi}=\lim _{\xi<\beta}\left(\alpha_{\xi}+1\right)=\alpha$ since $S$ is simple. Therefore (ii) is satisfied.

For $\xi<\beta$, let $\gamma_{\xi}$ be an ordinal such that $e_{0} \mathscr{D} e_{\gamma_{\xi}}$, and $\omega^{\alpha_{\xi}} \leqslant \gamma_{\xi}<\omega^{\alpha_{\xi}+1}$, and let $a_{\xi}$ be an element of $S$ such that $a_{\xi} a_{\xi}^{-1}=e_{0}$ and $a_{\xi}^{-1} a_{\xi}=e_{\gamma_{\xi}}$. Let $T_{\xi}$ be the subset of $S$ consisting of the elements $x \in S$ for which $e_{\omega^{\alpha} \xi}<\left(x x^{-1}\right)\left(x^{-1} x\right)$, together with the elements of the maximal subgroups containing the idempotents $e_{\zeta}$, $\zeta<\gamma_{\xi}$. If $\theta: S \rightarrow T_{\omega^{a}}, x \rightarrow\left(\begin{array}{l}\mu \\ \nu\end{array}\right)$, with $x x^{-1}=e_{\mu}$ and $x^{-1} x=e_{\nu}$ in $S$, stands for the canonical homomorphism of $S$ into the Munn semigroup $T_{\omega^{\alpha}}$, then $T_{\xi} \theta$ consists of the elements

$$
\left(\begin{array}{l}
\mu \\
\nu
\end{array}\right)=x \theta, \quad \text { with } x x^{-1}=e_{\mu}, \quad x^{-1} x=e_{\nu}, \quad \mu, \nu<\omega^{\alpha_{\xi}},
$$

and

$$
\left(\begin{array}{l}
\zeta \\
\zeta
\end{array}\right), \quad \zeta<\gamma_{\xi}
$$

Obviously $T_{\xi} \theta$ forms an inverse subsemigroup of the Munn semigroup $T_{\omega^{\alpha} \xi}$. Further, since $T_{\xi}=T_{\xi} \theta \theta^{-1}$, we deduce that $T_{\xi}$ forms an inverse subsemigroup of $S$. Let $S_{\xi}$ be the inverse subsemigroup of $S$ which is generated by $a_{\xi}$ and by the elements of $T_{\xi}$. Using Theorem 6 , we deduce that $S_{\xi}$ is (isomorphic to) a Bruck-Reilly extension of the $\gamma_{\xi}$-regular semigroup $T_{\xi}$, where $\gamma_{\xi}=\omega^{\alpha_{\xi}}+\delta_{\xi}$, with $\delta_{\xi}<\omega^{\alpha_{\xi}+1}$. Thus (i) is satisfied, and $S_{\xi}$ is a simple $\omega^{\alpha_{\xi}+1}$-regular semigroup (since $\left.\gamma_{\xi} \omega=\omega^{\alpha_{\xi}+1}\right)$.

We consider the system (11), where for $\xi \leqslant \eta<\beta, \phi_{\xi, \eta}: S_{\xi} \rightarrow S_{\eta}$ is just the inclusion mapping. We must show that $S$ is the direct limit of (11). Therefore, let $x$ be any element of $S$. Since $S$ is simple, there exists a $\gamma$ such that $e_{0} \mathscr{D} e_{\gamma}<$ $\left(x x^{-1}\right)\left(x^{-1} x\right)$. Let us suppose $\omega^{\alpha_{\xi}} \leqslant \gamma<\omega^{\alpha_{\xi}+1}$, with $\alpha_{\xi} \in A$. Then $x \in T_{\xi+1}$, and thus also $x \in S_{\xi+1}$. We conclude $S=\cup_{\xi<\beta} S_{\xi}$.

THEOREM 8. Let $T$ be a $\delta$-regular semigroup where $\omega^{\alpha} \leqslant \delta<\omega^{\alpha+1}$, and let $B R(T, \theta)$ be a Bruck-Reilly extension of $T$. Let e be an idempotent of $B R(T, \theta)$. Then eBR $(T, \theta)$ is a simple $\omega^{\alpha+1}$-regular semigroup.

Conversely, every simple $\omega^{\alpha+1}$-regular semigroup can be obtained in this way.

Proof. If $S$ is a simple regular semigroup, and $e \in E_{S}$, then $e S e$ is a simple regular subsemigroup of $S$. From this well-known fact follows the direct part of our theorem.

Let us conversely suppose that $S$ is a simple $\omega^{\alpha+1}$-regular semigroup. Let $E_{S}=\left\{e_{\xi} \mid \xi<\omega^{\alpha+1}\right\}$, where $e_{\xi}<e_{\eta}$ in $E_{S}$ if $\eta<\xi$. Let $D$ be the set of ordinals

$$
D=\left\{\xi \mid \xi=\eta-\zeta \geqslant \omega^{\alpha}, \omega^{\alpha+1}>\eta>\zeta, e_{\eta} \text { D } e_{\zeta} \text { in } S\right\},
$$


and let $\delta$ be the least ordinal in $D$. We have $\delta=\omega^{\alpha} n+\mu$, with $\mu<\omega^{\alpha}$. Let $\zeta$ and $\eta$ be any ordinals, with $\zeta<\eta<\omega^{\alpha+1}$, such that $e_{\eta} D e_{\zeta}$ in $S$ and $\eta-\zeta=\omega^{\alpha} n+\mu$. Putting $\zeta=\omega^{\alpha} m+\mu^{\prime}$, with $\mu^{\prime}<\omega^{\alpha}$, we have $\eta=\omega^{\alpha}(m+n)+\mu$. Let us investigate $S^{\prime}=e_{\zeta} S e_{\zeta}$.

$S^{\prime}$ is of course a $\omega^{\alpha+1}$-regular semigroup which is simple. Let $T$ be the subset of $S^{\prime}$ which consists of the elements of $S$ for which $e_{\eta}<x x^{-1}, x^{-1} x \leqslant e_{\zeta}$. Due to the minimality of $\delta$ in $D$, we have either

$$
e_{\zeta+\omega^{\alpha}(i-1)}<x x^{-1}, x^{-1} x \leqslant e_{\zeta+\omega^{\alpha} i}
$$

for some $i=\{0, \ldots, n-1\}$, or

$$
e_{\eta}<x x^{-1}, x^{-1} x \leqslant e_{\omega^{\alpha}(m+n)} .
$$

Take any other $y \in T$. Again, either

$$
e_{\zeta+\omega^{\alpha}(j+1)}<y y^{-1}, y^{-1} y \leqslant e_{\zeta+\omega^{\alpha} j}
$$

for some $j \in\{0, \ldots, n-1\}$, or

$$
e_{\eta}<y y^{-1}, y^{-1} y \leqslant e_{\omega^{a}(m+n)} .
$$

If $x$ and $y$ are elements of $T$ such that (12) and (14) or (15) hold, with $j>i$, then $x y \mathscr{K} y$, and so $x y \in T$. Similarly, if (14) and (12) or (13) hold, with $i>j$, then $x y \mathscr{K} x$, and thus $x y \in T$. Further, if (12) and (14) hold, with $i=j$, then

$$
e_{\zeta+\omega^{\alpha}(i+1)}<(x y)(x y)^{-1},(x y)^{-1}(x y) \leqslant e_{\zeta+\omega^{\alpha} i}
$$

and again $x y \in T$. Finally, let $x, y \in T$ such that both (13) and (15) hold. Let us suppose that $x y \notin T$, that is,

$$
e_{\omega^{a}(n+m+1)}<\left((x y)(x y)^{-1}\right)\left((x y)^{-1}(x y)\right)=e_{\nu} \leqslant e_{\eta} .
$$

Anyway, $x y \Re e_{\nu}$ or $x y \mathscr{L} e_{\nu}$, and $x y \mathscr{L} y$ or $x y \Re x$, since $E_{S}$ is a chain. Since both (13) and (15) hold, we conclude that there exists an idempotent $e_{\tau} \in E_{S}$, with $e_{\eta}<e_{\tau} \leqslant e_{\omega^{\alpha}(m+n)}$, such that $e_{\tau} \mathscr{D} e_{\nu}$. Let $\kappa=\nu-\eta$. Then $\kappa<\omega^{\alpha}$. If $a$ is any

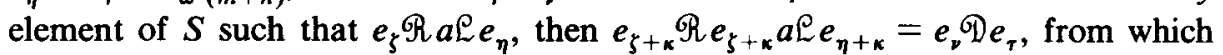
$e_{\zeta+\kappa} \mathcal{D} e_{r}$. Yet, $\tau-(\zeta+\kappa)<\delta$, since $\omega^{\alpha}(m+n) \leqslant \tau<\eta$, and this contradicts the minimality of $\delta$. Hence, also in this case $x y \in T$. We conclude that $T$ is a subsemigroup of $S^{\prime}$. It follows from Theorem 6 that $S^{\prime}$ is (isomorphic to) a Bruck-Reilly extension of $T$.

The identity $e_{0}$ of $S$ is $\mathscr{D}$-related to an idempotent $e_{\lambda}<e_{\zeta}$ since $S$ is simple. Let $b$ be any element of $S$ such that $b b^{-1}=e_{0}$ and $b^{-1} b=e_{\lambda}$. The mapping

$$
S \rightarrow e_{\lambda} S e_{\lambda}, \quad x \rightarrow b^{-1} x b,
$$

is an isomorphism of $S$ onto $e_{\lambda} S e_{\lambda}$. Yet $e_{\lambda} S e_{\lambda}=e_{\lambda} S^{\prime} e_{\lambda}$, where $S^{\prime}$ is (isomorphic to) a Bruck-Reilly extension $B R(T, \theta)$ of the $\delta$-regular semigroup $T$, with $\omega^{\alpha} \leqslant \delta$ $<\omega^{\alpha+1}$. From this follows the converse part of our theorem. 
COROllary 9 (Koč [4], Munn [8]). An inverse semigroup $S$ is a simple w-regular semigroup if and only if $S$ is a Bruck-Reilly extension of a finite chain of groups.

Not every simple $\omega^{\alpha+1}$-regular semigroup needs to be a Bruck-Reilly extension of a $\delta$-regular semigroup, with $\omega^{\alpha} \leqslant \delta<\omega^{\alpha+1}$. We depict a counterexample in Figure 1. Indeed, if $a$ is the element of the semigroup depicted in Figure 1 for

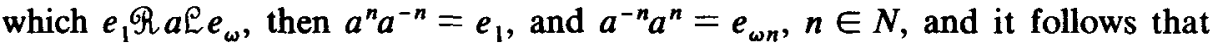
the subsemigroup requirement of Theorem 6(ii) cannot be satisfied. The inverse semigroup under consideration is a combinatorial simple $\omega^{2}$-regular semigroup. Remark however, that every bisimple $\omega^{\alpha+1}$-regular semigroup is a Bruck-Reilly extension of a $\omega^{\alpha}$-regular semigroup which is bisimple.

\section{Conclusion}

We note that we are now able to construct inductively all inverse semigroups with idempotents dually well-ordered. The process for doing so is based on Corollary 2, Theorem 7 and Theorem 8 . The techniques involved are the constructions of ordinal sums, direct limits and Bruck-Reilly extensions.

\section{The combinatorial case}

We conclude with some remarks concerning combinatorial inverse semigroups with idempotents dually well-ordered.

LEMMA 10. For any prime ordinal $\omega^{\beta}$, let $n\left(\omega^{\beta}\right)$ denote the number of pairwise non-isomorphic combinatorial simple $\omega^{\beta}$-regular semigroups. Then $\alpha<\beta$ implies $n\left(\omega^{\alpha}\right) \leqslant n\left(\omega^{\beta}\right)$.

Proof. Let $S$ be a combinatorial simple $\omega^{\alpha}$-regular semigroup, where $\alpha<\beta$. We may suppose that $S$ is a full inverse subsemigroup of $T_{\omega^{\alpha} \text {. The mapping }}$ $T_{\omega^{\alpha}} \rightarrow T_{\omega^{\beta}},\left(\begin{array}{l}\xi \\ \eta\end{array}\right) \rightarrow\left(\begin{array}{l}\xi \\ \eta\end{array}\right)$ is an embedding of $T_{\omega^{\alpha}}$ into $T_{\omega^{\beta}}$. Hence, we may suppose that $S$ is a subsemigroup of $T_{\omega^{\beta}}$, where $S$ consists of elements $\left(\begin{array}{l}\xi \\ \eta\end{array}\right)$, with $\xi, \eta<\omega^{\alpha}$. Let $S^{\prime}$ be the inverse subsemigroup of $T_{\omega^{\beta}}$ generated by the elements of $S$ and by the elements $\left(\begin{array}{c}0 \\ \omega^{n}\end{array}\right)$, where $\alpha \leqslant \nu<\beta$. Clearly $S^{\prime}$ is a combinatorial simple $\omega^{\beta}$-regular semigroup. 

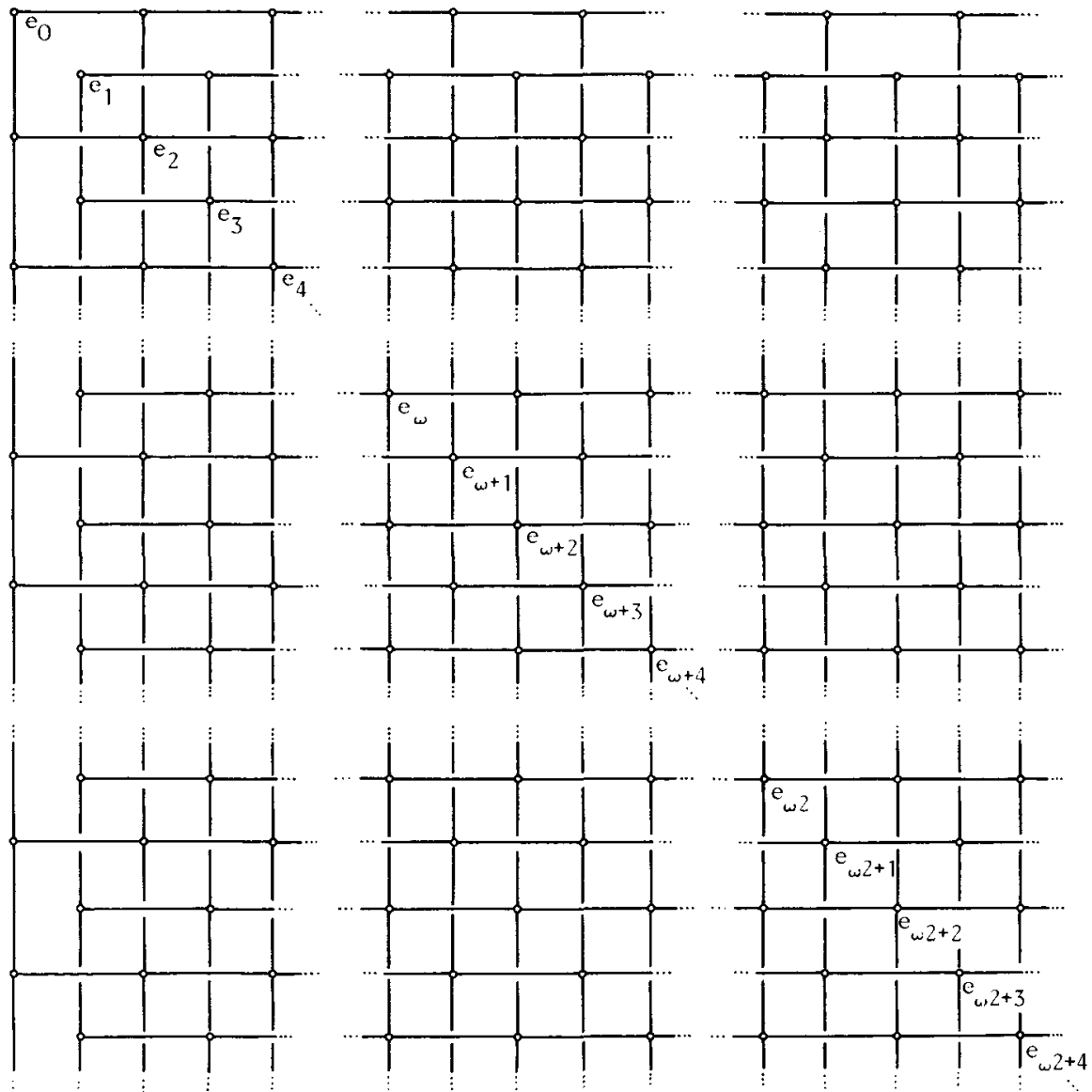

Figure 1

If $S_{1}$ and $S_{2}$ are two non-isomorphic combinatorial simple $\omega^{\alpha}$-regular semigroups, then $S_{1}^{\prime}$ and $S_{2}^{\prime}$ are non-isomorphic combinatorial simple $\omega^{\beta}$-regular semigroups. In other words, if we start off with a set of $n\left(\omega^{\alpha}\right)$ pairwise non-isomorphic combinatorial simple $\omega^{\alpha}$-regular semigroups, we obtain a set of pairwise non-isomorphic combinatorial simple $\omega^{\beta}$-regular semigroups. Thus $n\left(\omega^{\alpha}\right) \leqslant$ $n\left(\omega^{\beta}\right)$.

For any ordinal $\alpha, \omega_{\alpha}$ will denote an initial ordinal. In the following we assume the generalized continuum hypothesis. 
THEOREM 11. Let $\omega^{\beta}$ be a prime ordinal, and let $n\left(\omega^{\beta}\right)$ denote the number of pairwise non-isomorphic combinatorial simple $\omega^{\beta}$-regular semigroups. Then

$$
\begin{gathered}
n(\omega)=n\left(\omega^{2}\right)=\aleph_{0}, \\
n\left(\omega^{\beta}\right)=\aleph_{1} \quad \text { if } \omega^{3} \leqslant \omega^{\beta}<\omega_{1}, \\
n\left(\omega^{\beta}\right)=\aleph_{\alpha+1} \quad \text { if } \omega_{\alpha} \leqslant \omega^{\beta}<\omega_{\alpha+1}, \alpha \geqslant 1 .
\end{gathered}
$$

Proof. The result $n(\omega)=\boldsymbol{\aleph}_{0}$ follows easily from the results by Kočin [4] and Munn [8] (see also Petrich [10]). In fact one shows that the number of pairwise non-isomorphic combinatorial $\omega$-semigroups is $\boldsymbol{\aleph}_{0}$. Therefore also, if $\omega \leqslant \delta<\omega^{2}$, then there are only $\boldsymbol{\kappa}_{0}$ pairwise non-isomorphic combinatorial $\delta$-regular semigroups. From Theorem 8 one now deduces $n\left(\omega^{2}\right)=\boldsymbol{\aleph}_{0}$.

Every combinatorial $\omega^{\beta}$-regular semigroup can be embedded as a full inverse subsemigroup in $T_{\omega^{\beta}}$. If $\omega_{\alpha} \leqslant \omega^{\beta}<\omega_{\alpha+1}$, then $\left|T_{\omega^{\beta}}\right|=\kappa_{\alpha}$, thus also

$$
n\left(\omega^{\beta}\right) \leqslant \aleph_{\alpha+1} \text { if } \omega_{\alpha} \leqslant \omega^{\beta}<\omega_{\alpha+1} .
$$

Let us consider a mapping $f: N \rightarrow\{0,1\}$. Let us consider the system

$$
\left(\omega ;\left\{S_{\xi} \mid \xi<\omega\right\} ;\left\{\phi_{\xi, \eta} \mid \xi \leqslant \eta<\omega\right\}\right)
$$

where

(i) $S_{\xi} \cap S_{\eta}=\varnothing$ whenever $\xi \neq \eta$,

(ii) $S_{\xi}$ is a copy of the bicyclic semigroup whenever $f(\xi)=1$, and $S_{\xi}$ is a chain of order type $\omega^{*}$ whenever $f(\xi)=0$,

(iii) $\phi_{\xi, \eta}$ maps $S_{\xi}$ onto the identity of $S_{\eta}$ if $\xi<\eta<\omega$,

(iv) $\phi_{\xi, \xi}$ is the identity transformation on $S_{\xi}$ for $\xi<\omega$.

The sum of the system (17) is denoted by $S_{f}$. If $g: N \rightarrow\{0,1\}$ is any other mapping, with $f \neq g$, then $S_{f}$ is not isomorphic to $S_{g}$. In other words, we are able to construct $2^{\aleph_{0}}=\aleph_{1}$ pairwise non-isomorphic combinatorial $\omega^{2}$-regular semigroups. Using the method of constructing Bruck-Reilly extensions, we are able to construct $\aleph_{1}$ pairwise non-isomorphic combinatorial simple $\omega^{3}$-regular semigroups. Thus, by Lemma $10 n\left(\omega^{\beta}\right) \geqslant \boldsymbol{\aleph}_{1}$ whenever $\omega^{3} \leqslant \omega^{\beta}<\omega_{1}$. Using (16), we have $n\left(\omega^{\beta}\right)=\aleph_{1}$ whenever $\omega^{3} \leqslant \omega^{\beta}<\omega_{1}$.

Let us now consider an initial ordinal $\omega_{\alpha}\left(=\omega^{\omega_{\alpha}}\right), \alpha \geqslant 1$. Let $A_{1}\left[A_{2}\right]$ stand for the set of ordinals $\xi<\omega_{\alpha}$, which are of the form $\xi=\zeta+n$, with $n$ odd [even], and where the least primitive remainder of $\zeta$ does not equal 1 . Then $\omega_{\alpha}=A_{1} \cup A_{2}$, and $A_{1}$ and $A_{2}$ both constitute well-ordered chains of order type $\omega_{\alpha}$. Let $f$ : $A_{1} \rightarrow\{0,1\}$ by any mapping, and let $S_{f}$ be the full inverse subsemigroup of $T_{\omega_{\alpha}}$ 
which is generated by the elements

$$
\begin{gathered}
\left(\begin{array}{c}
\xi \\
\xi
\end{array}\right), \quad \xi<\omega_{\alpha}, \\
\left(\begin{array}{c}
0 \\
\omega^{\xi}
\end{array}\right), \quad \text { for all } \xi \in A_{2}, \\
\left(\begin{array}{c}
0 \\
\omega^{\xi}
\end{array}\right), \quad \text { for all } \xi \in A_{1} \text { for which } f(\xi)=1
\end{gathered}
$$

Then $S_{f}$ is a combinatorial simple $\omega_{\alpha}$-regular semigroup. Further, if $g: A_{1} \rightarrow\{0,1\}$ is any other mapping, with $g \neq f$, then $S_{f}$ cannot be isomorphic to $S_{g}$. Thus we constructed $\kappa_{\alpha+1}$ pairwise non-isomorphic combinatorial simple $\omega_{\alpha}$-regular semigroups. Using Lemma 10 , we see that for all $\omega_{\alpha} \leqslant \omega^{\beta}<\omega_{\alpha+1}$, we have $n\left(\omega^{\beta}\right) \geqslant$ $\aleph_{\alpha+1}$. Yet, by (16) we also have $n\left(\omega^{\beta}\right) \leqslant \aleph_{\alpha+1}$ and thus the equality $n\left(\omega^{\beta}\right)=$ $\aleph_{\alpha+1}$ prevails.

THEOREM 12. Let $S$ be a combinatorial simple $\omega^{\alpha}$-regular semigroup. The greatest group homomorphic image of $S$ is trivial if and only if a is a limit ordinal. Otherwise the greatest group homomorphic image of $S$ is the infinite cyclic group.

Proof. We may assume that $S$ is a full inverse subsemigroup of $T_{\omega^{\alpha}}$. Assume that $\alpha$ is a limit ordinal and let $\left(\begin{array}{l}\xi \\ \eta\end{array}\right) \in S$. Then $\xi, \eta<\omega^{\beta}<\omega^{\alpha}$ for some $\beta<\alpha$. So $\left(\begin{array}{l}\xi \\ \eta\end{array}\right)\left(\begin{array}{c}\omega_{\omega^{\beta}}^{\beta} \\ )\end{array}\right)=\left(\begin{array}{c}\omega^{\beta} \\ \omega^{\beta}\end{array}\right)\left(\begin{array}{l}\xi \\ \eta\end{array}\right)=\left(\begin{array}{c}\omega_{\omega^{\beta}}^{\beta} \\ )\end{array}\right)$, and we see that $S \times S$ is the least group congruence on $S$.

If $\alpha$ is not a limit ordinal, then $\alpha=\beta+1$ for some $\beta$. On $S$ we may now introduce a relation $\rho$ by

$$
\begin{array}{r}
\left(\begin{array}{l}
\xi \\
\eta
\end{array}\right) \rho\left(\begin{array}{l}
\xi^{\prime} \\
\eta^{\prime}
\end{array}\right) \quad \text { if and only if } \omega^{\beta} m \leqslant \xi, \xi^{\prime}<\omega^{\beta}(m+1) \text { and } \\
\omega^{\beta} n \leqslant \eta, \eta^{\prime}<\omega^{\beta}(n+1) \text { for some } m, n \in N .
\end{array}
$$

One may verify that $\rho$ is a congruence relation, and that $S / \rho$ is a combinatorial simple $\omega$-regular semigroup. If $\left(\begin{array}{l}\xi \\ \eta\end{array}\right) \rho\left(\begin{array}{l}\xi^{\prime} \\ \eta^{\prime}\end{array}\right)$ as in (18), and if $k=\max (m, n)+1$, then

$$
\left(\begin{array}{c}
\omega^{\beta} k \\
\omega^{\beta} k
\end{array}\right)\left(\begin{array}{l}
\xi \\
\eta
\end{array}\right)\left(\begin{array}{c}
\omega^{\beta} k \\
\omega^{\beta} k
\end{array}\right)=\left(\begin{array}{c}
\omega^{\beta} k \\
\omega^{\beta} k
\end{array}\right)\left(\begin{array}{c}
\xi^{\prime} \\
\eta^{\prime}
\end{array}\right)\left(\begin{array}{c}
\omega^{\beta} k \\
\omega^{\beta} k
\end{array}\right),
$$

which implies that $\left(\begin{array}{l}\xi \\ \eta\end{array}\right)$ and $\left(\begin{array}{l}\xi^{\prime} \\ \eta^{\prime}\end{array}\right)$ are related in the least group congruence on $S$. Thus, the greatest group homomorphic image of $S$ coincides with the greatest homomorphic image on $S / \rho$, that is, it is the infinite cyclic group. 


\section{References}

[1] E. H. Feller and R. L. Gantos, 'Completely injective semigroups', Pacific J. Math. 31 (1969), 359-366.

[2] J. Hogan, 'Bisimple semigroups with idempotents well-ordered', Semigroup Forum 6 (1973), 298-316.

[3] J. M. Howie, An introduction to semigroup theory (Academic Press, London, 1976).

[4] B. P. Kočin, 'Structure of inverse ideally-simple $\omega$-semigroups', Vestnik Leningrad. Univ. Mat. 23, nr. 7 (1968), 41-50 (in Russian).

[5] L. Megyesi and G. Pollák, 'Uber die Struktur der Hauptidealhalbgruppen. I', Acta Sci. Math. (Szeged) 29 (1968), 261-270.

[6] L. Megyesi and G. Pollák, 'Uber die Struktur der Hauptidealhalbgruppen. Il', Acta Sci. Math. (Szeged) 39 (1977), 103-108.

[7] L. Megyesi and G. Pollák, 'On simple principal ideal semigroups', Studia Math., to appear.

[8] W. D. Munn, 'Regular w-semigroups', Glasgow Math. J. (1968), 46-66.

[9] W. D. Munn, personal communication.

[10] M. Petrich, Structure of regular semigroups (Cahiers Mathématiques, Université des Sciences et Techniques du Languedoc, Montpellier, 1977).

[11] N. R. Reilly, 'Bisimple w-semigroups', Proc. Glasgow Math. Assoc. 7 (1966), 160-167.

[12] W. Sierpinski, Cardinal and ordinal numbers (Polish Scientific Publishers, Warszawa, 1965).

[13] R. J. Warne, 'A class of bisimple inverse semigroups', Pacific J. Math. 18 (1966), 563-577.

[14] R. J. Warne, 'Bisimple inverse semigroups mod groups', Duke Math. J. 34 (1967), 787-812.

[15] G. L. White, 'The dual ordinal of a bisimple inverse semigroup', Semigroup Forum 6 (1973), 295-297.

\section{Dienst Hogere Meetkunde}

Rijksuniversiteit Gent

Krijgslaan 281

B-9000 Gent

Belgium 